\title{
Electronic Structure of Hydrogenated and Surface-Modified GaAs Nanocrystals: Ab Initio Calculations
}

\author{
Hamsa Naji Nasir, ${ }^{1}$ Mudar A. Abdulsattar, ${ }^{2}$ and Hayder M. Abduljalil1 \\ ${ }^{1}$ Physics Department, College of Science, University of Babylon, Babylon, Iraq \\ ${ }^{2}$ Directorate of materials research, Ministry of Science and Technology, Baghdad, Iraq \\ Correspondence should be addressed to Mudar A. Abdulsattar, mudarahmed3@yahoo.com
}

Received 9 October 2012; Revised 19 November 2012; Accepted 9 December 2012

Academic Editor: R. N. P. Choudhary

Copyright (C) 2012 Hamsa Naji Nasir et al. This is an open access article distributed under the Creative Commons Attribution License, which permits unrestricted use, distribution, and reproduction in any medium, provided the original work is properly cited.

\begin{abstract}
Two methods are used to simulate electronic structure of gallium arsenide nanocrystals. The cluster full geometrical optimization procedure which is suitable for small nanocrystals and large unit cell that simulates specific parts of larger nanocrystals preferably core part as in the present work. Because of symmetry consideration, large unit cells can reach sizes that are beyond the capabilities of first method. The two methods use ab initio Hartree-Fock and density functional theory, respectively. The results show that both energy gap and lattice constant decrease in their value as the nanocrystals grow in size. The inclusion of surface part in the first method makes valence band width wider than in large unit cell method that simulates the core part only. This is attributed to the broken symmetry and surface passivating atoms that split surface degenerate states and adds new levels inside and around the valence band. Bond length and tetrahedral angle result from full geometrical optimization indicate good convergence to the ideal zincblende structure at the centre of hydrogenated nanocrystal. This convergence supports large unit cell methodology. Existence of oxygen atoms at nanocrystal surface melts down density of states and reduces energy gap.
\end{abstract}

\section{Introduction}

GaAs bulk or nanocrystals are excellent materials in many electronic devices [1-4]. The direct band gap of GaAs gives it superiority in light-emitting devices. Nanocrystals of GaAs are manufactured in different methods that range from laser ablation [2] to chemical implantation [4]. These nanocrystals give us the ability to maneuver many physical properties such as energy gap and lattice constant to fulfill the range of requirements of electronic devices.

In the present work we shall investigate some properties of GaAs nanocrystals using two different ab initio methods. The first method is the usual cluster full geometrical optimization in connection with Hartree-Fock method (HF) which is one of the most accurate methods to simulate electronic structure of nanocrystals. This method is also the most computationally expensive both in time and resources [57]. The second method is the large unit cell method (LUC) which was developed recently from previous applications to bulk solids [8-14]. The LUC method was initially associated with semiempirical methods such as the intermediate neglect of differential overlap (LUC-INDO) due to computational time difficulties of the last century and the beginning of the present one $[8,9,12,13]$. At present most LUC calculations are performed using ab initio methods $[10,11,14]$. The periodic boundary condition (PBC) utility that is essential for performing LUC calculations are available to run with DFT in the Gaussian 03 program $[5,6]$ which is used in the present work.

\section{Theory}

Two different ab initio molecular orbital methods are used in the present work to simulate the electronic structure of gallium arsenide nanocrystals. The first method is the cluster geometrical optimization method (Figure 1) that uses Hartree-Fock theory with STO-3G basis to complete their molecular orbital calculations. The above basis and method is used to allow the reach to higher number of atoms. This 
method includes the variation of all atomic positions until we arrive to a nearly zero forces (with a given tolerance) acting on all atoms in the nanocrystal in their stationary positions $[5,6]$. The second method is the large unit cell method (LUC) which can be used to simulate systems of periodical symmetry such as the bulk or surfaces of ordinary crystals. The LUC is adopted for the simulation of the electronic structure of nanocrystals in conjunction with the $\mathbf{k}=0$ approximation ( $\mathbf{k}$ is the reciprocal wave vector). This approximation is used to drop sums of contributions from other points in $\mathbf{k}$ space except the origin [10-14]. This is translated in nanocrystals structure by saying that we have a limited translational symmetry in the inside "core" of the nanocrystal only (Figure 2). Since the inside core of the nanocrystal has a well-defined 3D symmetry structure such as in the present zincblende structure, we optimize the structure by optimizing the lattice constant of the inner core only. This method can be used to simulate nanocrystals surfaces by adopting the $\mathbf{k}=0$ approximation but with more elaborate optimization procedure. Surface simulation procedure includes the optimization of interlayer distances (perpendicular to the surface) in addition to the lattice constant since we have a 2D symmetry at the surface $[10,11,14-16]$. Density functional theory at the generalizedgradient approximation level coupled with large unit cell method (LUC-DFT) is used to simulate the electronic structure of zincblende gallium arsenide nanocrystals.

Four LUC cores are considered in the present work 8, 16, 54, and 64 atoms [17]. The 8 atom LUC (for example) is the core part of a larger nanocrystal that has 216 atoms after periodically repeating the 8 atom LUC in 3D. Some of these atoms surround and passivated outer dangling bonds of the central 8 atom core LUC. Although this core part is nearly constant and stable in its electronic structure, the surface part might be variable because of surface orientation, shape, reconstruction and different kind of passivating atoms such as hydrogen, oxygen, and so forth. The time needed for the evaluation of the electronic structure of this part is much smaller than the time needed for the full geometrical optimization procedure because of the ideal structure and symmetry consideration used in LUC method.

\section{Calculations and Results}

GaAs nanocrystal cluster of stoichiometry $\mathrm{Ga}_{31} \mathrm{As}_{32} \mathrm{H}_{69}$ is investigated in the present work using full geometrical optimization as in Figure 1. The size of this nanocrystal $(1.48 \mathrm{~nm})$ is chosen carefully so that four layers are between the surface and the centre of the nanocrystal in addition to the hydrogen atoms passivating layer. This size of nanocrystal is an ideal size for nanocrystal calculations since it is a compromise between size and computational efforts using present computational facilities. After full geometrical optimization the lattice constant is $5.45 \AA$ as estimated from bonds lengths at the center of the nanocrystal. The energy gap of this nanocrystal is $9.57 \mathrm{eV}$ and the valence band width is $19.51 \mathrm{eV}$. Figure 3 shows the variation of tetrahedral angle between atoms starting from arsenide hydrogenated

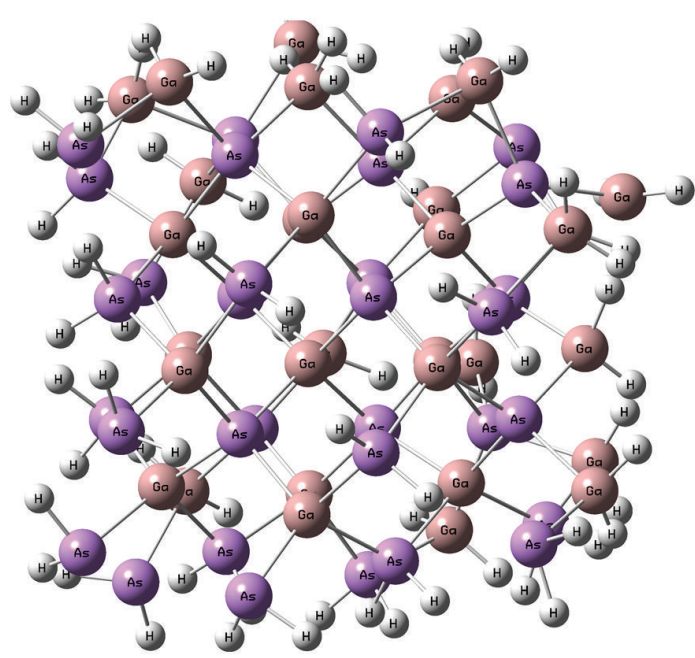

Figure 1: (Color online) Geometrically optimized hydrogenated $\mathrm{GaAs}$ nanocrystal cluster of the stoichiometry $\mathrm{Ga}_{31} \mathrm{As}_{32} \mathrm{H}_{69}$.

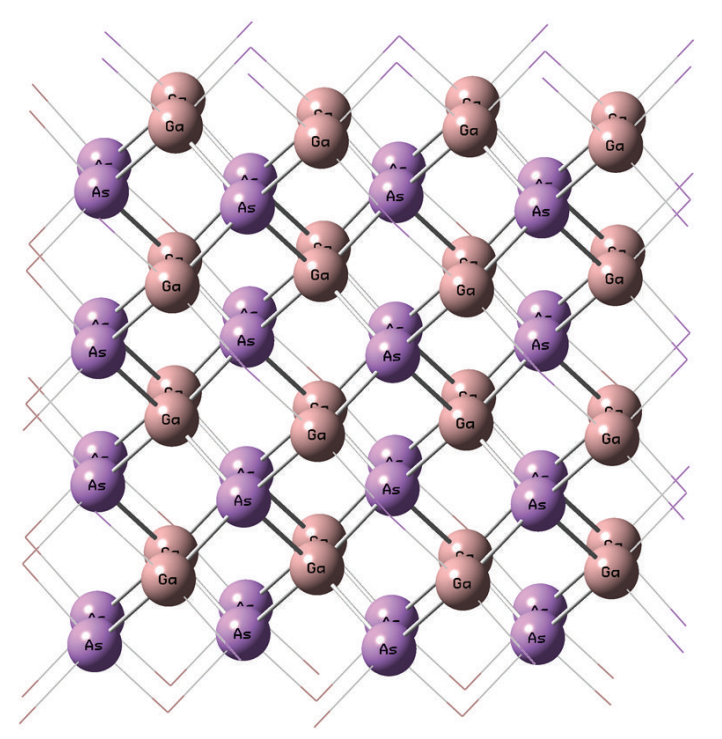

FIGURE 2: (Color online) 64 atoms at the core of GaAs nanocrystal as viewed by the LUC method.

surface until we reach the gallium hydrogenated surface layer at the opposite face of the nanocrystal. This angle is compared with the ideal diamond and zincblende structure tetrahedral angle of $109.47^{\circ}$. Figure 4 shows the bond lengths starting from arsenide hydrogenated surface until we reach the gallium hydrogenated surface layer at the opposite face of the nanocrystal. Figure 5 shows density of states of hydrogenated GaAs nanocrystal cluster and hydrogenated GaAs nanocrystal with oxygen atom replacing two hydrogen surface atoms.

Unlike the full optimization procedure, the LUC optimization includes the variation of the lattice constant only for the core part of the nanocrystal. This procedure is repeated for the four considered LUCs 8, 16, 54, and 64 atoms. The sizes of these LUCs cover the range $2-2.52 \mathrm{~nm}$ 


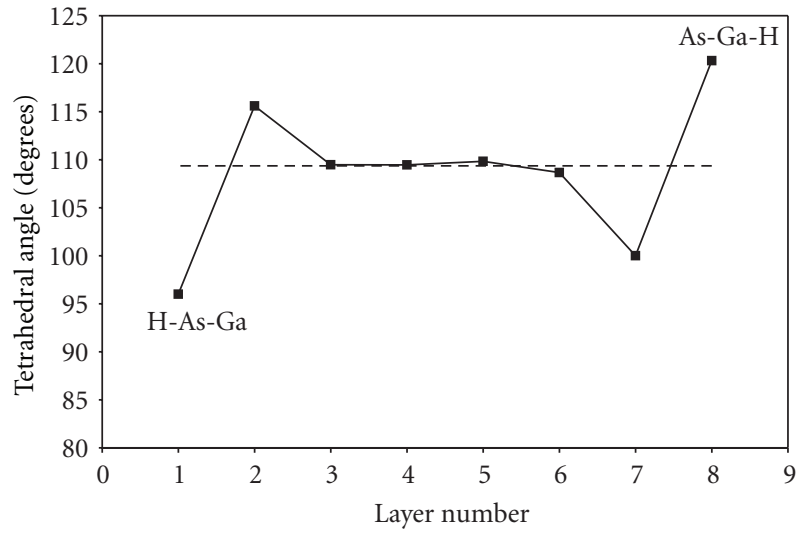

FIgURE 3: The variation of tetrahedral angle between atoms starting from the arsenide terminated surface (H-As-Ga angle) until we reach the gallium terminated layer (As- $\mathrm{Ga}-\mathrm{H}$ angle) at the opposite side of the nanocrystal (solid line) using cluster-HF geometrical optimization. This angle is compared with the ideal diamond and zincblende structure tetrahedral angle of $109.47^{\circ}$ (broken line).

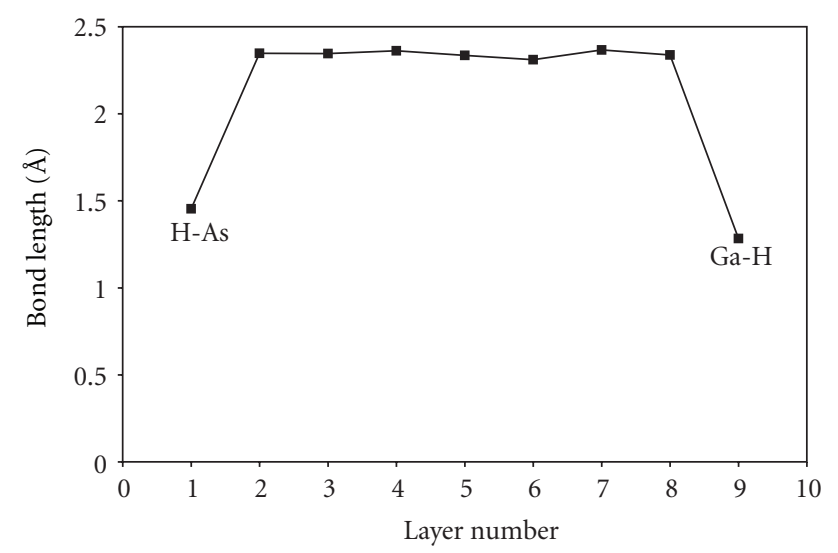

Figure 4: The variation of bond length between atoms starting from the arsenide hydrogenated surface ( $\mathrm{H}-\mathrm{As}$ bond) until we reach the tenth layer at the hydrogenated gallium surface ( $\mathrm{Ga}-\mathrm{H}$ bond) of the nanocrystal using cluster-HF geometrical optimization.

and the range of atoms 216 to 512 atoms in the total nanocrystal size that include the surface [17]. The optimized lattices are plotted against number of core atoms in Figure 6. The same is true for energy gap and valence band width in Figures 7 and 8 , respectively.

\section{Discussion and Conclusions}

The effect of surface on the total electronic structure decreases rapidly as nanocrystals grow up in size. The reason is that the size of surface reconstructed region is proportional to the square of the dimension of the nanocrystal while the size of core part is proportional to the cube of the dimension of the nanocrystal. This fact is one of important reasons behind LUC method that concentrates on the core part of the nanocrystal. In fact, surface reconstructed electronic features

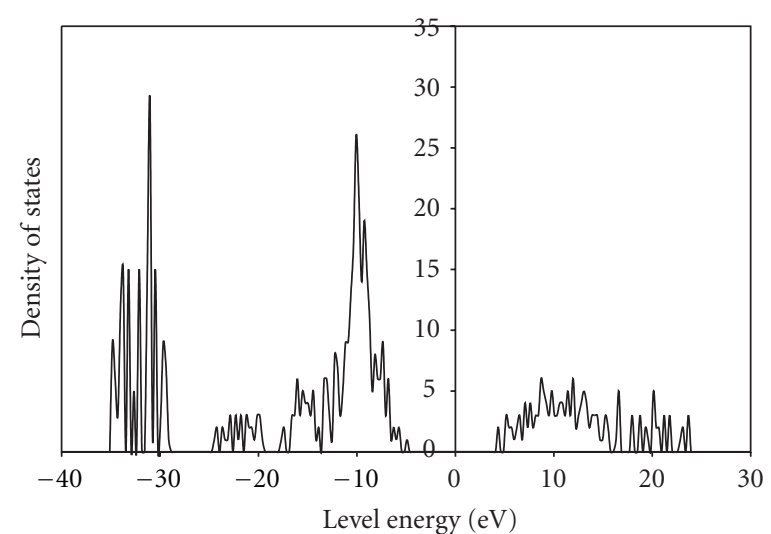

(a)

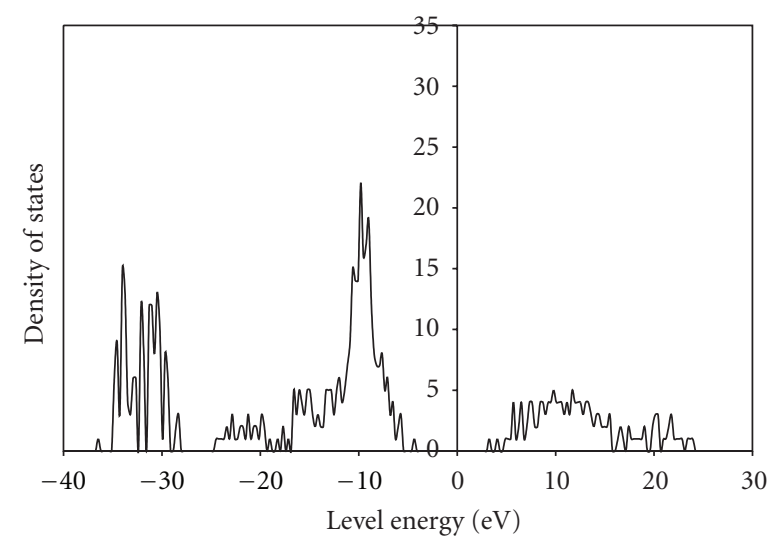

(b)

Figure 5: Density of states of hydrogenated GaAs nanocrystal cluster (a), and hydrogenated GaAs nanocrystal cluster with an oxygen atom replacing two hydrogen surface atoms (b). HartreeFock theory is used in the calculations. Density of states is collected in intervals of 0.01 Hartree $(0.27211 \mathrm{eV})$.

show themselves as shallow lines in comparison with the more intense lines of bulk [16].

Figure 3 shows the rapid convergence of tetrahedral angle to the ideal value of this angle in diamond and zincblende structures at the centre of the fully optimized $\mathrm{Ga}_{31} \mathrm{As}_{32} \mathrm{H}_{69}$ nanocrystal. This convergence is in agreement with the known impression that surface reconstruction are mainly at the outer three layers $[15,16]$. This fast convergence is a supporting evidence of the correctness of LUC hypothesis that the inner core of nanocrystals are nearly at the ideal structure positions after four layers. We can also note from Figure 3 that surface reconstruction reaches deeper layers at the gallium hydrogenated surface (right side of Figure 3 ) than that of arsenide side (left side of Figure 3). The difference between these two elements, metallic Ga element and semimetal As behavior is the reason behind this phenomenon which are observed at the bulk surfaces as well [18].

Figure 4 shows that As-Ga bond variation is limited inside the nanocrystal within a small tolerance in the fully optimized $\mathrm{Ga}_{31} \mathrm{As}_{32} \mathrm{H}_{69}$ nanocrystal. This figure also shows 


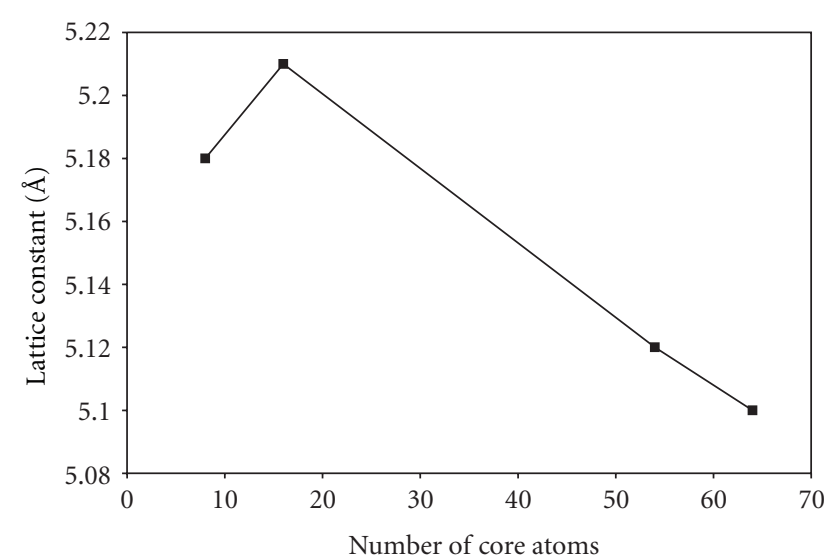

FIGURE 6: Lattice constant variation as a function of number of core atoms in LUC-DFT method for GaAs nanocrystals.

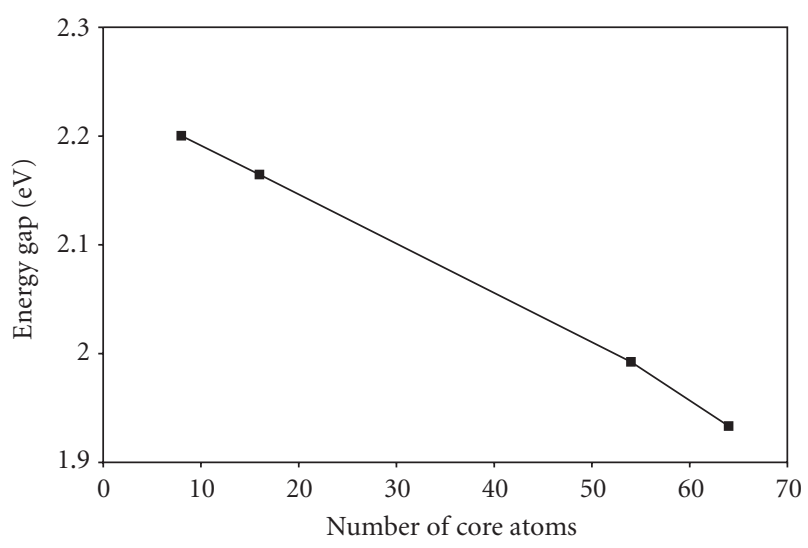

Figure 7: Energy gap variation as a function of number of core atoms in LUC-DFT method for GaAs nanocrystals.

that $\mathrm{As}-\mathrm{H}$ and $\mathrm{Ga}-\mathrm{H}$ bonds have the lengths 1.45 and $1.28 \AA$ respectively at the present selected path. Bond variation at the Ga terminated surface is slightly more than that of As terminated surface. This is also attributed to the differences between the two elements as in the case of the tetrahedral angle.

Figure 5 shows the density of states of the fully optimization GaAs nanocrystal with and without oxygen atom replacing two hydrogen surface atoms. The density of states seems to experience a melting of the high density peaks upon oxidation. This also includes the reduction of the band gap and widening of both valance and conduction bands. Previous theoretical and experimental results for bulk GaAs crystals showed that the $1.42 \mathrm{eV}$ gap vanishes due to surface oxidation [18]. This result is in good agreement with the present reduction of the gap due to oxidation from 9.57 to $7.81 \mathrm{eV}$ (i.e., $1.76 \mathrm{eV}$ ).

Figure 6 shows the variation of the lattice constant with the number of core atoms in LUC method. This (general) lattice reduction as nanocrystals grow up in size had been observed in all IV and some III-V semiconductors theoretically $[10,11,13,14,17,19]$ and experimentally in diamond [20]. The increase of lattice constant between 8 and 16 atom

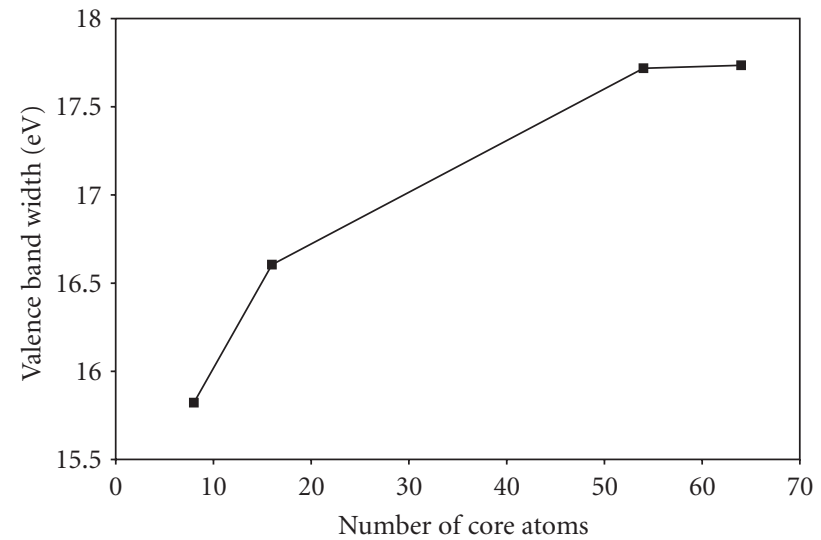

FIGURE 8: Valence band width variation as a function of number of core atoms in LUC-DFT method for GaAs nanocrystals.

LUC is due to shape effects since 8 atom LUC is a Bravais lattice while 16 atom LUC is a primitive lattice multiple [17]. The lattice constant at the centre of the cluster-HF full geometrically optimized nanocrystal of Figure $1(5.45 \AA)$ is larger than that of Figure 6 lattice constant values using LUCDFT method. This is expected since the GaAs nanocrystal of the stoichiometry $\mathrm{Ga}_{31} \mathrm{As}_{32} \mathrm{H}_{69}$ is smaller in size than all LUCs of the present work. Experimentally, the lattice constant of bulk GaAs is $5.65 \AA$ [1]. This value is in acceptable agreement with the value obtained using full geometrical optimization $(5.45 \AA)$ taking into consideration correlation correction that are not added in the present work. The further approximations used in LUC method are expected to lead to additional deviation from the experimental value as can be seen in Figure 6.

Figure 7 shows a decreasing order of the energy gap as a function of LUC number of atoms. This decrease is in agreement with the usual trend of nanocrystals that is imposed by quantum confinement theory. However, the comparison with the gap of the fully optimized hydrogenated nanocrystal $(9.57 \mathrm{eV})$ reveals that the energy gap reduction is very much more rapid than that observed for the lattice constant. In fact this fast reduction is supported by previous experimental and theoretical results [3]. All the calculated gaps in the present work for the two methods are blue shifted with respect to the bulk gap experimental value at $1.42 \mathrm{eV}$ [1].

Figure 8 shows that the valence band width of the GaAs LUC increases and converges to a value slightly below $18 \mathrm{eV}$. Unlike the previous properties, the valence band width of the hydrogenated nanocrystal is not nearer to the 8 atom LUC but is higher than all LUCs at the value of $19.51 \mathrm{eV}$. This contradiction is in fact is due to the surface reconstruction layer in the fully optimized nanocrystal. Surface reconstruction always widens valance and conduction bands. This is a direct result of broken symmetry and new kind of atoms that passivated the dangling bonds at the surface (hydrogen atoms in the present case). The new atoms and broken symmetry introduces new levels inside the energy gap and around both valence and conduction bands. The highly degenerate states 
at the core of the nanocrystal split at the surface and increases the valence band $[10,11,17]$.

As concluding remarks from the above discussion, we observe that the two methods are complementary in their results. The trend of the lattice constant from the two present methods indicates a slight reduction of the lattice constant as the size of the nanocrystal increases. On the other hand the energy gap encounters large reduction in its value as we go from the small size simulated by full geometrical optimization to the larger sizes simulated by LUC method. Unlike the above two properties, the valence band width of nanocrystals using full geometrical optimization is out of the trend encountered in LUC method. The inclusion of surface in full geometrical optimization greatly widens the valence band width that surpasses all LUC calculated values. The broken symmetry at the surface and surface passivating atoms is the cause of such large increase in valence band width. Bond length and tetrahedral angel results from cluster full geometrical optimization indicate good convergence to the ideal zincblende structure at the centre of hydrogenated nanocrystal with the convergence to be faster at the arsenic terminated surface. This convergence is in favor and supports LUC methodology. Oxygen atoms at the nanocrystal surface melt down density of states and reduce the energy gap of the nanocrystal.

\section{References}

[1] S. M. Sze and K. K. Ng, Physics of Semiconductor Devices, John Wiley \& Sons, 3rd edition, 2007.

[2] J. Perrière, E. Millon, M. Chamarro, M. Morcrette, and C. Andreazza, "Formation of GaAs nanocrystals by laser ablation," Applied Physics Letters, vol. 78, no. 19, pp. 29492951, 2001.

[3] J. Jiang, B. Gao, T. T. Han, and Y. Fu, "Ab initio study of energy band structures of GaAs nanoclusters," Applied Physics Letters, vol. 94, no. 9, Article ID 092110, 2009.

[4] S. Okamoto, Y. Kanemitsu, K. S. Min, and H. A. Atwater, "Photoluminescence from GaAs nanocrystals fabricated by $\mathrm{Ga}^{+}$and $\mathrm{As}^{+}$co-implantation into $\mathrm{SiO}_{2}$ matrices," Applied Physics Letters, vol. 73, no. 13, pp. 1829-1831, 1998.

[5] J. B. Foresman and A. E. Frisch, Exploring Chemistry with Electronic Structure Methods: A Guide to Using Gaussian, Gaussian, 2nd edition, 1996.

[6] M. J. Frisch, G. W. Trucks, and H. B. Schlegel, Gaussian 03, Revision B.01, Gaussian, Pittsburgh, Pa, USA, 2003.

[7] M. A. Abdulsattar, "SiGe superlattice nanocrystal pure and doped with substitutional phosphorus single atom: density functional theory study," Superlattices and Microstructures, vol. 50, p. 377, 2011.

[8] A. H. Harker and F. P. Larkins, "A large unit cell semiempirical molecular orbital approach to the properties of solids. I. General theory," Journal of Physics C, vol. 12, no. 13, article 013, pp. 2487-2495, 1979.

[9] I. O. Radi, M. A. Abdulsattar, and A. M. Abdui-Lettif, "Semiempirical LUC-INDO calculations on the effect of pressure on the electronic structure of diamond," Physica Status Solidi B, vol. 244, no. 4, pp. 1304-1317, 2007.

[10] N. H. Aysa, M. A. Abdulsattar, and A. M. Abdul-Lettif, "Electronic structure of germanium nanocrystals core and
(001)-(1×1) oxidised surface," Micro and Nano Letters, vol. 6, no. 3, pp. 137-140, 2011.

[11] M. A. Abdulsattar, "Mesoscopic fluctuations of electronic structure properties of boron phosphide nanocrystals," Electronic Materials Letters, vol. 6, no. 3, pp. 97-101, 2010.

[12] M. A. Abdulsattar and K. H. Al-Bayati, "Corrections and parametrization of semiempirical large unit cell method for covalent semiconductors," Physical Review B, vol. 75, no. 24, Article ID 245201, 2007.

[13] M. A. Abdulsattar, "Size effects of semiempirical large unit cell method in comparison with nanoclusters properties of diamond-structured covalent semiconductors," Physica E, vol. 41, no. 9, pp. 1679-1688, 2009.

[14] H. M. Abduljalil, M. A. Abdulsattar, and S. R. Al-Mansoury, "SiGe nanocrystals core and surface electronic structure from Ab initio large unit cell calculations," Micro \& Nano Letters, vol. 6, p. 386, 2011.

[15] C. Kittel, Introduction to Solid State Physics, John Wiley \& Sons, 7th edition, 1996.

[16] S. J. Sque, R. Jones, and P. R. Briddon, "Structure, electronics, and interaction of hydrogen and oxygen on diamond surfaces," Physical Review B, vol. 73, no. 8, pp. 1-15, 2006.

[17] M. A. Abdulsattar, "Ab initio large unit cell calculations of the electronic structure of diamond nanocrystals," Solid State Sciences, vol. 13, no. 5, pp. 843-849, 2011.

[18] H. Gang and S. Zhaoqi, High-k Gate Dielectrics for CMOS Technology, chapter 13, John Wiley \& Sons, 2012.

[19] H. R. Jappor, Z. A. Saleh, and M. A. Abdulsattar, "Simulation of electronic structure of aluminum phosphide nanocrystals using ab initio large unit cell method," Advances in Materials Science and Engineering, vol. 2012, Article ID 180679, 6 pages, 2012.

[20] C. Wen, Z. H. Jin, X. X. Liu et al., "Studies on nano-diamond prepared by explosive detonation by Raman and infrared spectroscopy," Spectroscopy and Spectral Analysis, vol. 25, no. 5, pp. 681-684, 2005. 

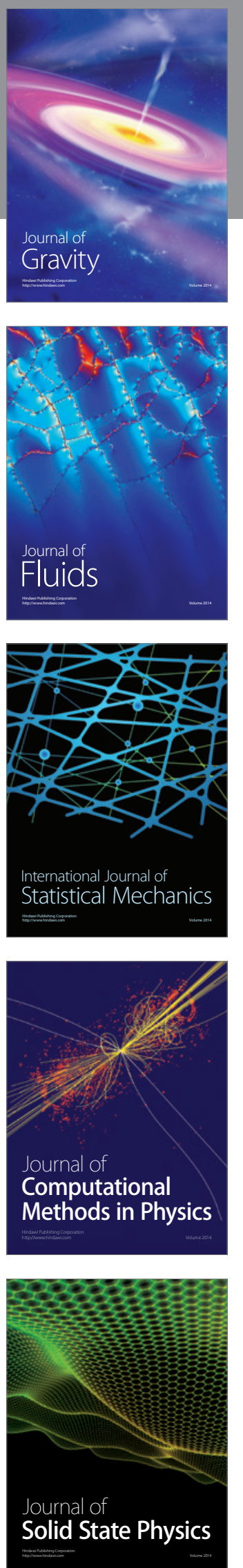

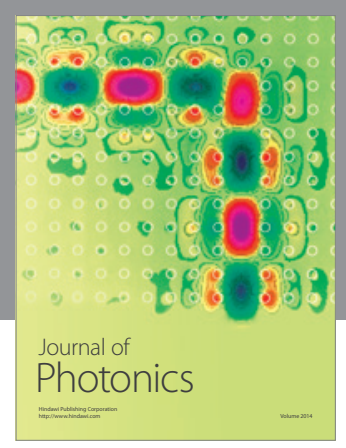

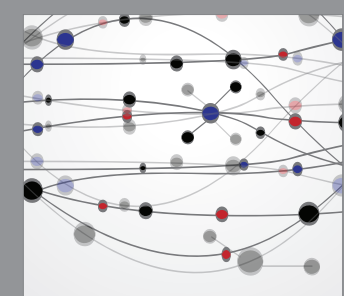

The Scientific World Journal
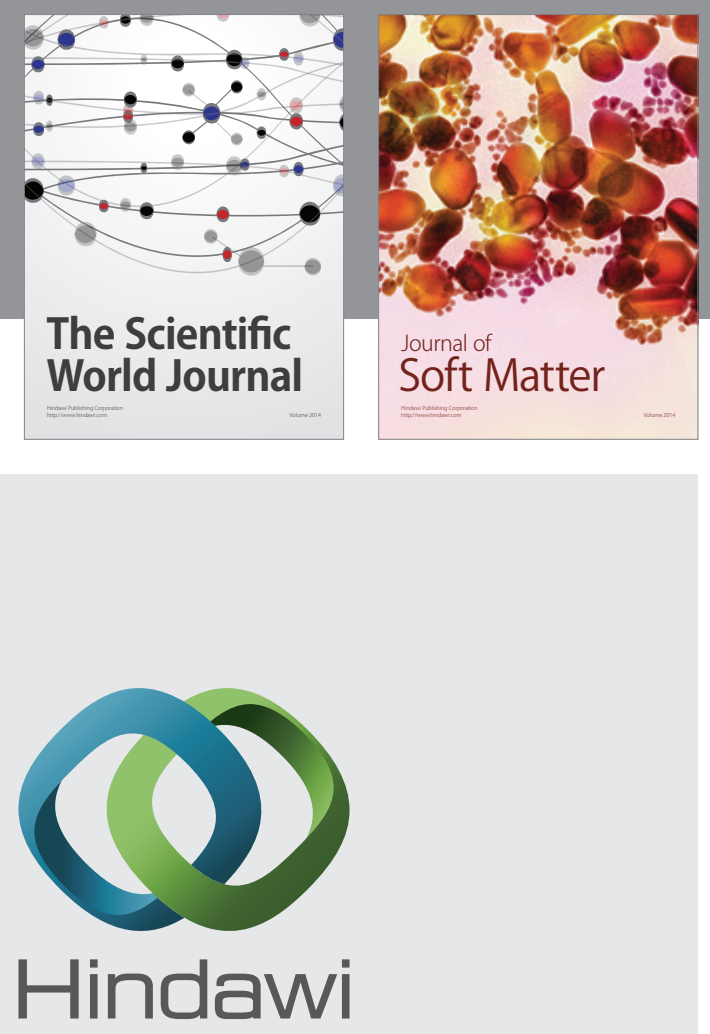

Submit your manuscripts at

http://www.hindawi.com
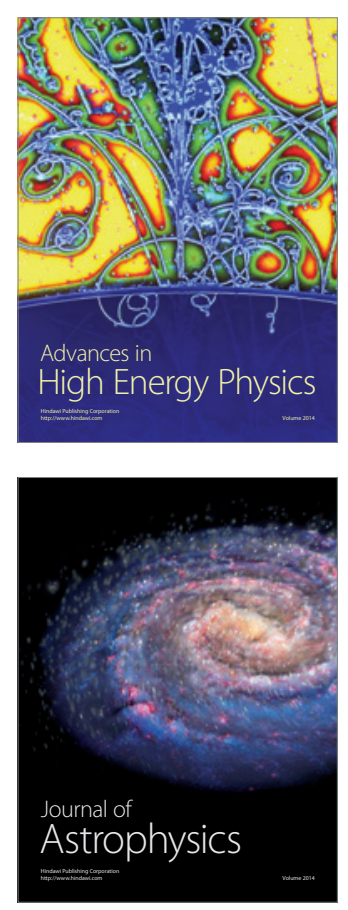
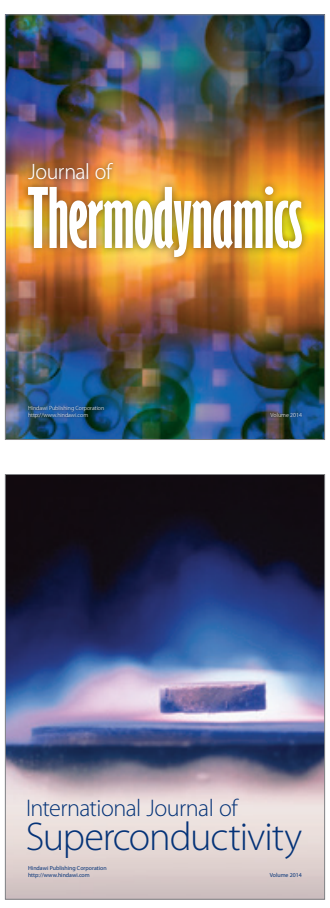
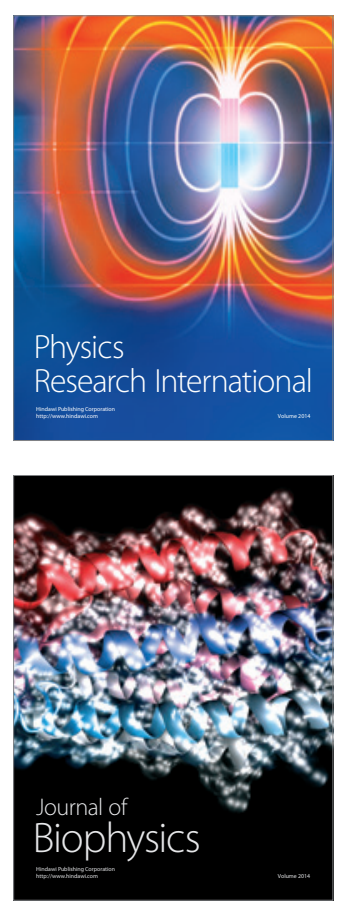
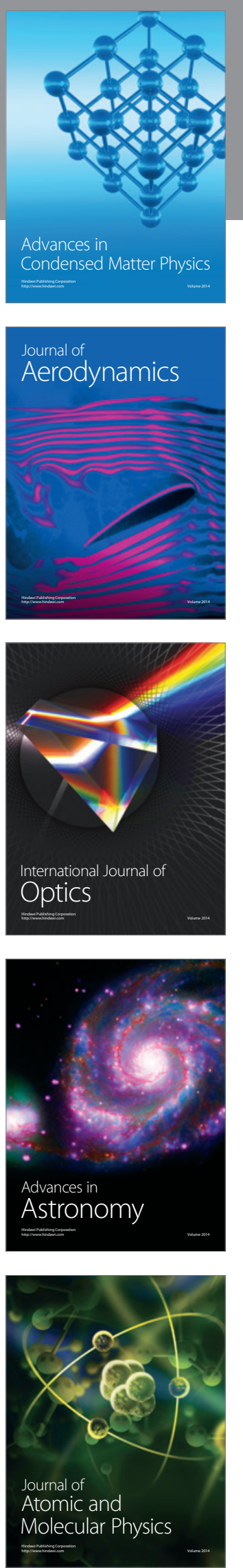